\title{
Degradation of electrooptical characteristics of serial GaP light-emitting diodes, caused by fast electrons
}

\author{
O.V. Konoreva ${ }^{1}$, M.V. Lytovchenko ${ }^{1}$, Ye.V. Malyi ${ }^{1}$, I.V. Petrenko ${ }^{1}$, M.B. Pinkovska ${ }^{1}$, \\ V.P. Tartachnyk ${ }^{1}$, V.V. Shlapatska ${ }^{2}$ \\ ${ }^{I}$ Institute for Nuclear Research, NAS of Ukraine, 47, prospect Nauky, 03028 Kyiv, Ukraine \\ ${ }^{2}$ L. Pisarzhevsky Institute of Physical Chemistry, NAS of Ukraine, \\ 31, prospect Nauky, 03028 Kyiv, Ukraine \\ Corresponding author: phone+38(044)-525-37-49; e-mail: evgen.malyj@gmail.com
}

\begin{abstract}
The study of electrical and optical characteristics of GaP LEDs irradiated with electrons $\left(E=2 \mathrm{MeV}, \Phi=0 \ldots 10^{15} \mathrm{~cm}^{-2}\right)$ was performed. Especial interest was focused on appearing of $S$-type instability in current-voltage characteristics and its mechanism in accord with the model by K. Maeda. Made in this work was the analysis of changes in the concentration of deep recombination levels caused be irradiation. The coefficient of radiation damages of charge carrier lifetime in $\mathrm{GaP}$ was estimated $\left(k_{\tau}=1.5 \cdot 10^{7} \mathrm{~s} \cdot \mathrm{cm}^{-2}\right)$. It was discovered that the luminescence intensity recovering after isochronous annealing possesses two stages. Heating the diodes up to $T>350{ }^{\circ} \mathrm{C}$ leads to destruction of $p$-region in the diode and deterioration of lens optical transmission.
\end{abstract}

Keywords: gallium phosphide, light-emitting diode, defects, electron irradiation, currentvoltage characteristics, annealing.

Manuscript received 30.03.15; revised version received 18.06.15; accepted for publication 03.09.15; published online 30.09.15.

\section{Introduction}

Modern light-emitting diodes (LEDs) are useful in a wide spectrum - from infrared to ultraviolet, and their use is constantly expanding. Now one can talk about diode's effective operation in the field of precision instrumentation and in the manufacture of household appliances. Compared with all other types of sources of previous generations, LEDs possess significant advantages - low power consumption, miniature, speed, great exploitation resource, reliability and low cost. However, under the influence of extreme external factors - ionizing radiation, temperature and others diodes change characteristics and degrade. Often, this process is uncontrolled and irreversible, leading to failures in control systems. Deviations are especially harmful in the optoelectronic circuits of control and regulating devices at NPP or communication lines Earth-Space.

Irradiation influence on GaP LEDs was studied in a number of works [1-5], but some unresolved questions concerning the nature of nonradiative levels caused by irradiation, the damage coefficients, the position of the main recovery stages, change of the transparency of lens coatings and others still exist.

Here, we have presented the result of our researches aimed at serial green and red diodes, irradiated with $2-\mathrm{MeV}$ electrons. The main attention has been devoted to the study of current-voltage properties and recovering the diode parameters after annealing. 


\section{Experimental}

Serial light-emitting GaP diodes were fabricated by double liquid-epitaxy. $n$-type substrate doped by Te to the concentration $n \sim 10^{17} \mathrm{~cm}^{-3}$ was cut from grown $\mathrm{Cz}$ $\mathrm{GaP}$ ingot. Epitaxial layer of red diodes was additionally doped with Te in the $n$-region and with zinc and oxygen simultaneously - in the $p$-region.

Current-voltage characteristics and electrominescence spectral curves were measured by the automatic equipment within 77 to $300 \mathrm{~K}$ temperature interval. Samples were irradiated with $2-\mathrm{MeV}$ electrons to the fluences $(0 \ldots 1.3) \cdot 10^{15} \mathrm{~cm}^{-2}$ in the pulsed mode from the accelerator, and temperature didn't exceed $300 \mathrm{~K}$. Annealing was carried out isochronously with the 20-min period.

\section{Results and discussion}

The main peculiarity of current-voltage characteristics of the diodes is the existence of the region with the negative differential resistance (NDR) at low temperatures (Fig. 1). It appears during measurements in the mode of current generator.

Under conditions $\left|R_{d}^{-}\right|<R_{L}$, and $R_{L}>\frac{L}{\left|R_{d}^{-}\right| C}$, where $R_{d}$ is the resistance of the sample and $R_{L}$ is the resistance of the loading, $L, C$ are the parameters of the measuring system, $S$-type region of NDR desintegrates into a number of oscillations with the oscillation frequency of a few kilohertz (Fig. 2).

In the mode of voltage generator, the region of sharp current increase appears (Fig. 1, point A) instead of the $S$-type curve.

The Schockly equation [6] for current $I\left(U_{p-n}\right)$ through $p-n$ junction is as follows:

$$
I=I_{S}\left(e^{\frac{q U_{p-n}}{m k T}}-1\right),
$$

where $I_{s}$ is the saturation current, $m-$ constant $(m=$ $1 \ldots 2)$.

At a low level of injection of minority carriers through the $p-n$ junction, when there is no modulation of the base resistance, and $R_{b}$ is constant, the voltage drop in the depleted region is equal to

$U_{p-n}=\frac{m k T}{q} \ln \frac{I+I_{S}}{I_{S}}$.

The resistance of $p$ - $n$ junction

$$
R_{p-n}=\frac{d U_{p-n}}{d I}=\frac{m k T}{q} \frac{1}{I+I_{S}} .
$$

Thus, $R_{p-n}$ decreases with current growth.

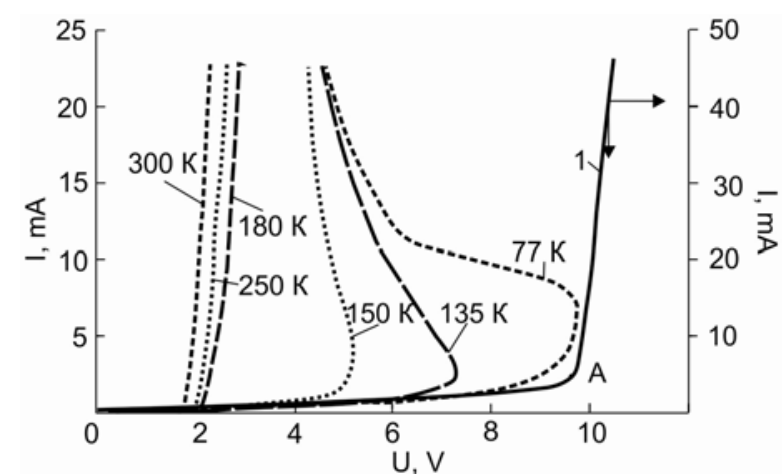

Fig. 1. Current-voltage characteristics of initial GaP LEDs in the current (at different temperatures) and voltage (1) generator modes.

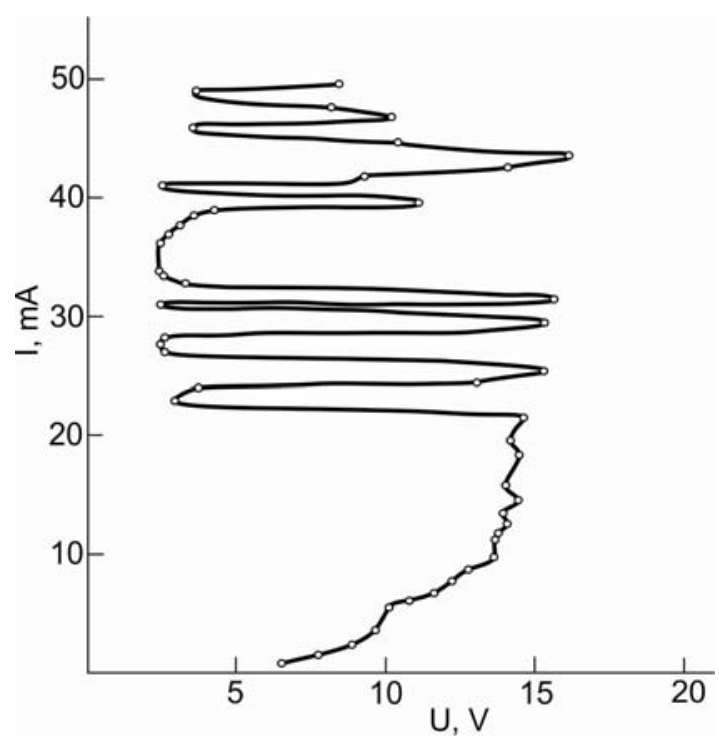

Fig. 2. Current-voltage characteristics of $\mathrm{GaP}$ diodes for $\left|R_{d}^{-}\right|<R_{L}$ and $R_{L}>\frac{L}{\left|R_{d}^{-}\right| C}$ at $77 \mathrm{~K}$.

The diode voltage drop $U$ consists of a sum of voltages at the base and $p-n$ junction

$U=U_{b}+U_{p-n}=I R_{b}+\frac{m k T}{q} \ln \frac{1}{I+I_{S}}$.

From the experiment it is clear that in the point of diode current transition to the NDR mode (Fig. 1, point A) $\frac{d U}{d t}=0$. When $U=U_{A}, I \rightarrow \infty$, and Eq. (1) is inapplicable.

Change in the carrier transport mechanism through the sample begins when $I(U)$ exponential dependence, valid for low currents $\left(I<10^{-5} \mathrm{~A}\right)$, is broken. Further increase of injection level through the $p-n$ junction causes an internal inverse positive connection and formation of $S$-like region of negative differential resistance (NDR) of current-voltage characteristics. 
Depending on material there are different mechanisms responsible for non-monotonous $I(U)$. For example, Lampert and co-authors in paper [7] analyzed in detail the double currier injections in isolators. In his early work, Bhargava described the appearance of the negative resistance region in current-voltage characteristics of $\mathrm{GaP}$ [8]. Later K. Maeda [9] considered the influence of deep recombination levels in the high resistive $n$-area of GaP $n-p$ transition on the shape of before-breakdown region of current. It was stated that, if the concentration of centers was higher than that of free carriers, and asymmetry of capture cross-section was large enough $\left(\sigma_{p}>>\sigma_{n}\right)$, then one can describe beforebreakdown region of current as

$$
J=e \mu \mu_{n}^{2} \frac{\alpha V_{n}^{2}}{\sigma_{n} v L^{3}},
$$

where $J$ is the current density, $\alpha-$ constant $(\alpha<1), v-$ carrier thermal velocity, and $L-$ length of the highresistivity region, $V_{n}=U$.

Under given conditions, the current density is proportional to the square of the applied voltage $V_{n}$. It is the result of double carriers' injection into highresistivity region of diode, when increase of the current changes the occupancy of recombination levels.

The dependence $I(U)$ in logarithm coordinates of red $\mathrm{GaP}$ LEDs is given in Fig. 3. One can see that for both, initial $(77 \mathrm{~K})$ and irradiated $(150 \ldots 300 \mathrm{~K})$ samples, $I(U)$ is linear with the slope $2.26 \ldots 2.6$ (for different samples) when the voltage changes from $2.5 \ldots 3.0 \mathrm{~V}$ to $U_{w}$ (wrecking). That suggests the double injection mode. In the given region, the voltage increase gives rise of the drift current and provokes the appearance of the internal positive feedback. Then empty recombination levels, when $\sigma_{p}>>\sigma_{n}$, are filled with holes. The lifetime grows strongly, and holes are accumulated in the high-resistivity region, where there are recombination levels. The avalanche current appears, and $S$-instability takes place.

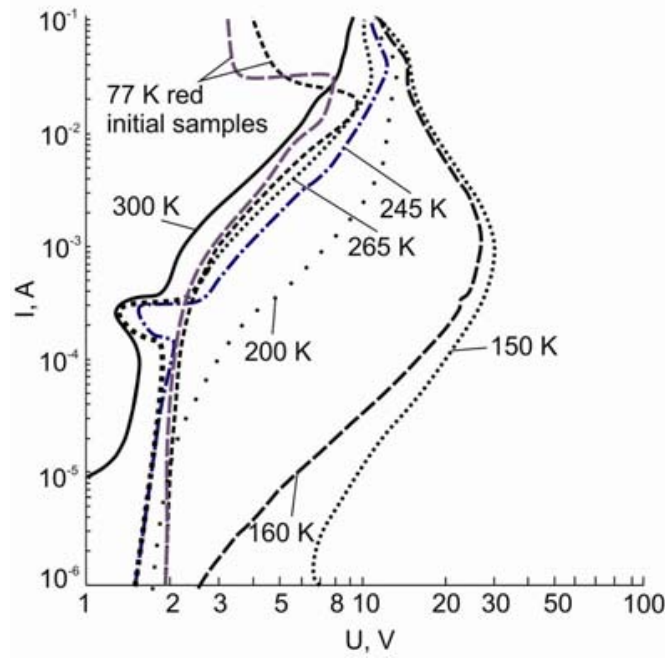

Fig. 3. Current-voltage characteristics of GaP LEDs: initial and irradiated with $2-\mathrm{MeV}$ electrons $\left(\Phi=8.2 \cdot 10^{16} \mathrm{~cm}^{-2}\right)$ at different temperatures.
Diode transit into the NDR mode begins when $U_{w}=U_{A}=V_{w}$

$V_{w}=\frac{L^{3} N \sigma_{n} \nu}{l_{0} \mu_{n}}\left(\frac{\sigma_{n}}{\sigma_{p}}\right)^{1 / 2}$,

where $N$ is the concentration of recombination levels and $l_{0}$ - diffusion length. The wrecking voltage $V_{w}$ depends on temperature (Fig. 3), it is caused by the change in the charge state of levels caused by the temperature shift of the Fermi level.

Diode irradiation leads to the $V_{w}$ growth caused by the increase in the concentration of deep level radiation defects and decrease in the carrier mobility $\mu_{n}$. The dependence of $\mu_{n}(T)$ for initial and irradiated samples is given in Fig. 4. One can see that at the nitrogen temperature irradiation causes the mobility drop by 1.55 times, and at the same time the breakdown voltage is almost 3-fold increased. Comparing the wrecking voltage $V_{w}$ for initial and irradiated diode (Eq. (6)), it is possible to consider the change in the concentration $N$ of deep levels caused by irradiation. For $10^{16} \mathrm{~cm}^{-2}$ fluence, the value $N$ grows twice.

Electroluminescence spectra of red and green diodes at room temperatures are the symmetrical structureless bands with the maxima $h v_{1}=1.797 \mathrm{eV}$ and $h v_{2}=2.22 \mathrm{eV}$, respectively. For nitrogen temperature electroluminescence spectra are shown in Fig. 5; their irradiation maxima are $h v_{1}=1.85 \mathrm{eV}$ for red and $h v_{2}=$ $2.275 \mathrm{eV}$ for green specimens. Electron irradiation leads to a monotonic decline in the luminescence intensity due to introduction of deep nonradiative levels of radiation defects.

The value of the diffusion current through the $p-n$ junction is determined, in addition to all other variables, by $\tau$ (lifetime of minority charge carriers) [6].

$I \sim \tau^{-\frac{1}{2}} e^{\frac{q U}{m k T}}$

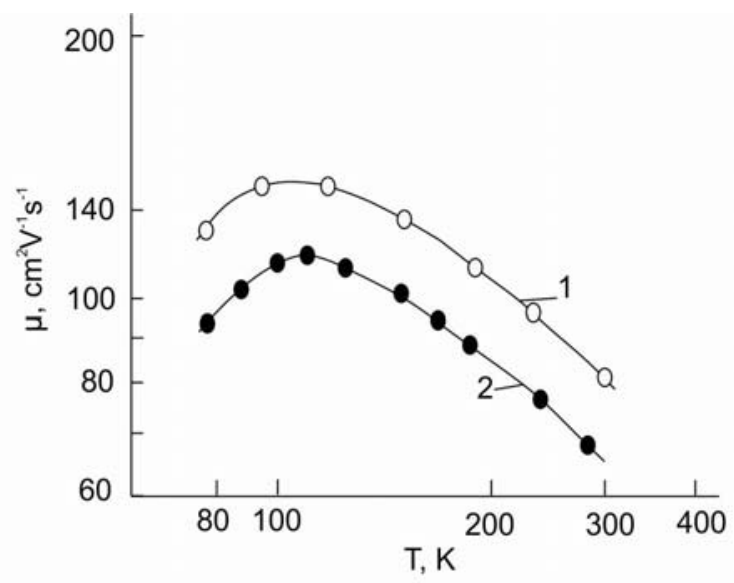

Fig. 4. Dependences of electron mobility of GaP LEDs on temperature: initial (1) and irradiated (2) with $1-\mathrm{MeV}$ electrons $\left(\Phi=8.2 \cdot 10^{16} \mathrm{~cm}^{-2}\right)$. 


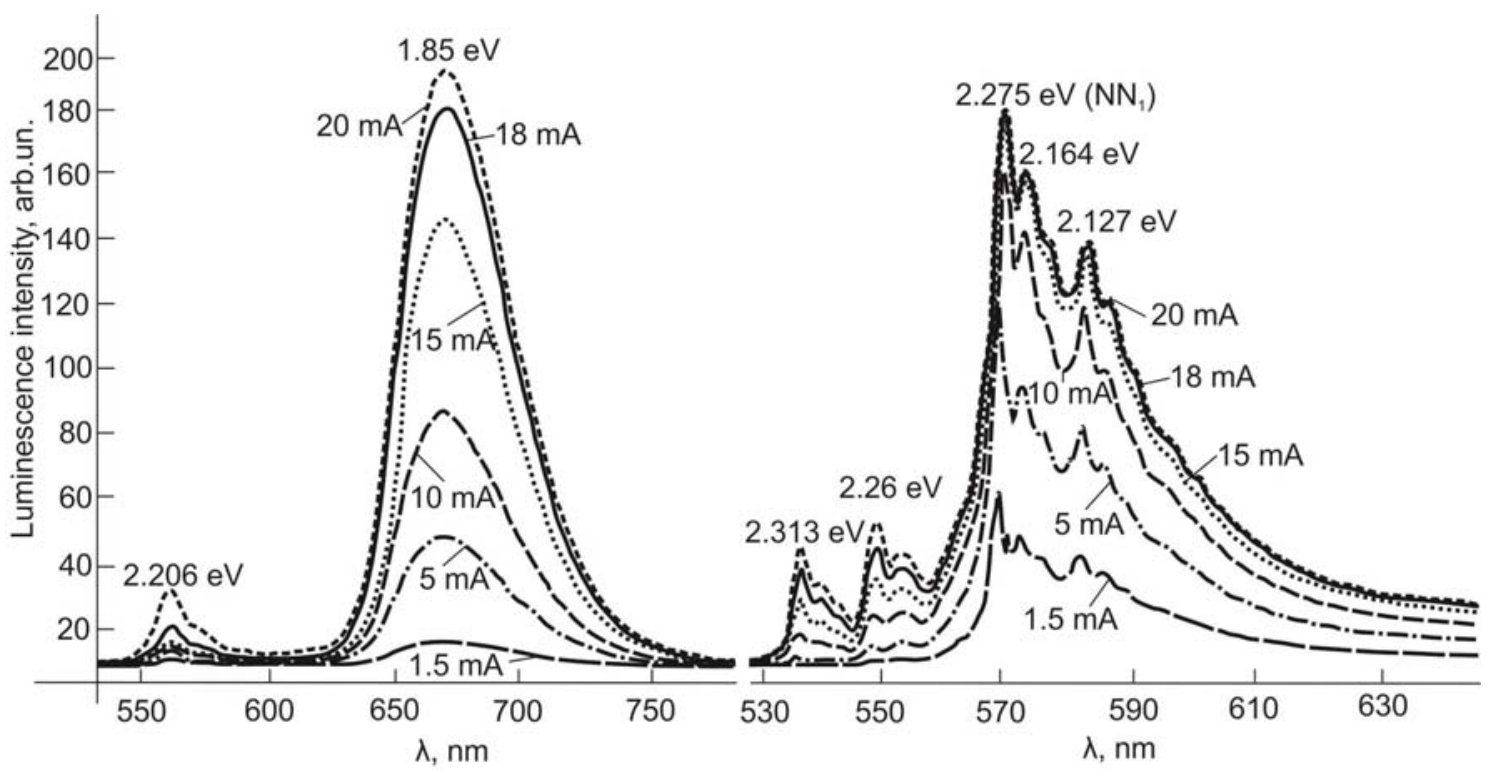

Fig. 5. Luminescence spectra of red (left side) and green (right side) initial GaP LEDs at different excitation levels at $T=77 \mathrm{~K}$.

On the other hand, the luminescence intensity

$L \sim \tau e^{\frac{q U}{m k T}}$,

then

$L_{0}=A \tau_{0}^{3 / 2} I$,

$A$ is the proportional coefficient and $\tau_{0}-$ lifetime of minority carriers in the initial diode.

For irradiated diode

$L=A \tau_{\Phi}^{3 / 2} I$,

then

$\frac{L}{L_{0}}=\left(\frac{\tau_{\hat{\mathrm{O}}}}{\tau_{0}}\right)^{\frac{3}{2}}$.

It is usefull to express $\tau_{\Phi}$ through the damage coefficient of carriers' lifetime [10]

$\frac{1}{\tau_{\Phi}}=\frac{1}{\tau_{0}}+\frac{\Phi}{k_{\mathrm{r} C}}$.

Substituting $\tau_{\Phi}$ into the formula (11), we obtain

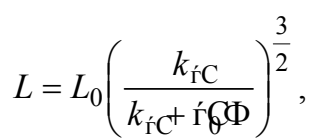

$\left(\frac{L_{0}}{L}\right)^{\frac{2}{3}}-1=\frac{\tau_{0}}{k_{\tau}} \Phi$.

This formula is analogous to the relation for the quantum efficiency in [10].
From the experimental data (Fig. 6), it is clear that the linear dependence $\Phi$ on $\left(\frac{L_{0}}{L}\right)^{\frac{2}{3}}-1$ is valid up to $\Phi \approx$ $6 \cdot 10^{14} \mathrm{~cm}^{-2}$ dose.

The slope of the curve makes it possible to determine the initial rate of radiation damage of minority carriers' lifetime for this type of irradiation. If the $\tau_{0}$ value is considered to be $10^{-8} \mathrm{~s}$, the coefficient of radiation damage of minority carriers' lifetime is equal $k_{\tau} \cong 1.5 \cdot 10^{7} \mathrm{~s} \cdot \mathrm{cm}^{-2}$.

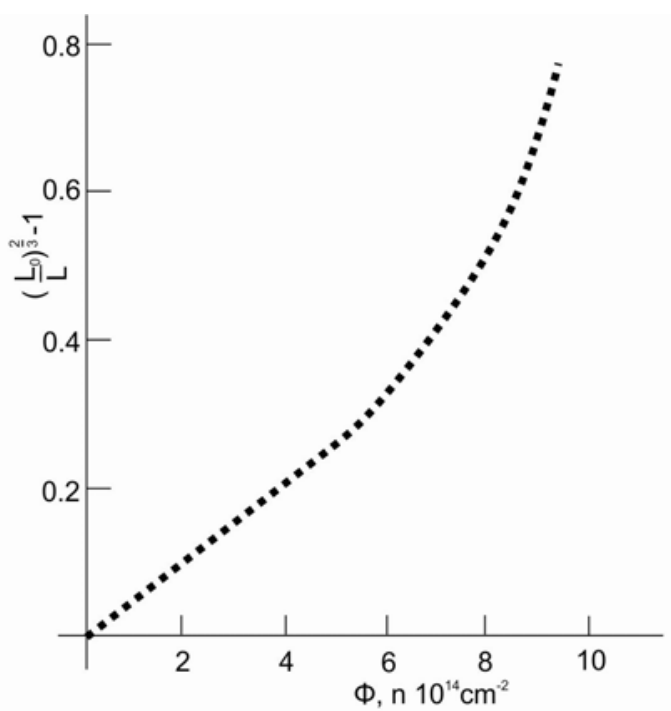

Fig. 6. Dependence of $\frac{L^{2 / 3}}{L_{0}}-1$ on the electron fluence $\Phi$ for GaP LEDs. 


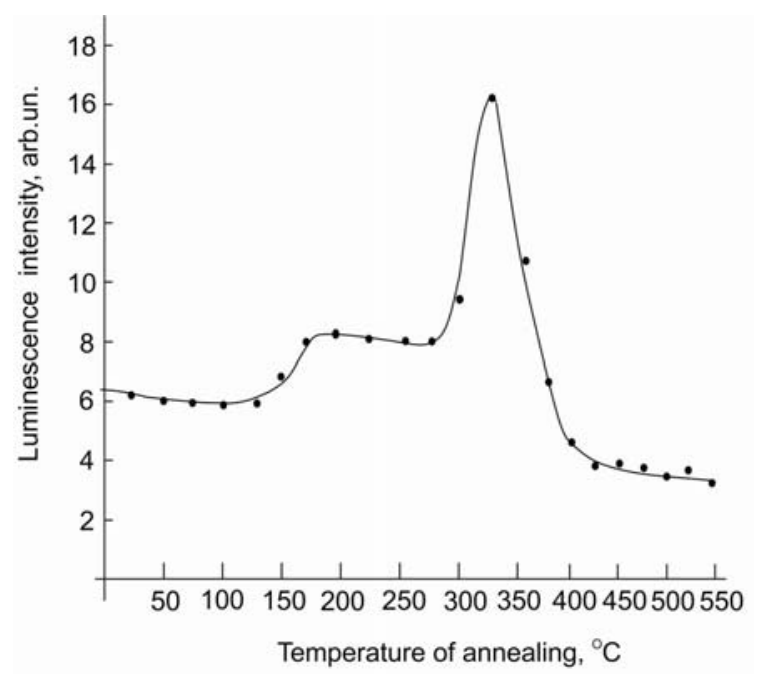

Fig. 7. Recovery of the luminescence intensity of red GaP LED irradiated with electrons in the process of isochronal annealing.

Fig. 7 shows the isochronal annealing curve of red $\mathrm{GaP}: \mathrm{Zn}, \mathrm{O}$ diode, irradiated with $2-\mathrm{MeV}$ electrons to $4.12 \cdot 10^{14} \mathrm{~cm}^{-2}$ fluence. One can see two ranges of recovering the emission intensity: $150 \ldots 200^{\circ} \mathrm{C}$ and $275 \ldots 325^{\circ} \mathrm{C}$. The former is a main stage for $n$-GaP, where $V_{p}$ is annealed; the second one is related with annealing of $\mathrm{V}_{\mathrm{Ga}}$ in the $p$-region of the diode [11]. This is a main stage in red diodes, because red luminescence appears in the $p$-region of the structure.

The drop of the luminescence intensity at $T>350{ }^{\circ} \mathrm{C}$ occurs due to the influence of two factors: a decrease in the optical transmittance of the diode lens and worsening the homogeneity of the $p$-conducting base, which was observed in [11]. It should be also noted that changing the transparency of the coating is accompanied by its "painting" - lens gradually becomes brown.

\section{Conclusion}

The region of negative differential resistance (NDR) appears in the current-voltage characteristics of $\mathrm{GaP}$ LEDs at low temperatures $(T \leq 90 \mathrm{~K})$. The effect depends on positive feedback guided by the current.

It is shown that the degradation curve of electroluminescence intensity allows determining the coefficient of radiation damages. For GaP diodes after $2-\mathrm{MeV}$ electron irradiation, it begins equal to $k_{\tau}=$ $1.5 \cdot 10^{7} \mathrm{~s} \cdot \mathrm{cm}^{-2}$.
The partial recovery of the radiation intensity in red LED GaP has two temperature stages: $150 \ldots 200^{\circ} \mathrm{C}$ and $275 \ldots 325^{\circ} \mathrm{C}$. After $T>350^{\circ} \mathrm{C}$, the luminescence intensity decreases monotonically due to increased levels of defects in the $p$-region of the diode after hightemperature annealing.

It was also found that the value of the optical transmission of lens coating material also decreases at temperatures $T>350{ }^{\circ} \mathrm{C}$.

\section{References}

1. A.A. Antoshin, F.P. Korshunov, S.M. Samsonov, Quenching of the individual electroluminescence bands of GaP:Zn,O LEDs under the action of fast electrons // Zhurnal prikladnoi spektroskopii, 40, No. 5, p. 758-760 (1984), in Russian.

2. I.A. Sukach, Radiation-induced transformation of radiative exciton complexes bound to nitrogen in GaP:N green light-emitting structures // J. Lumines. Mater. 8, p. 139-152 (1979).

3. V.S. Manzhara, V.P. Tartachnyk, Nature of negative differential resistance of $\mathrm{GaP}$ lightemitting diodes // Ukrainian Journal of Physics, 46, No.2, p. 191-195 (2001).

4. P. Litovchenko, D. Bisello, A. Litovchenko et al., Some features of current-voltage characteristics of irradiated GaP light diodes // Nucl. Instr. and Methods in Phys. Res. A, 552, p. 93-97 (2005).

5. O. Konoreva, B. Opilat, M. Pinkovska, V. Tartachnyk, Features of current-voltage characteristics inherent to GaP light-emitting diodes with quantum wells // Semiconductor Physics, Quantum Elecronics and Optoelectronics, 9, No.4, p. 45-48 (2006).

6. S. Sze, Physics of Semiconductor Devices, vol. 1. Moscow, Mir, 1984, p. 455 (in Russian).

7. M.A. Lampert and A. Rose, Volume-controlled, two-carrier currents in solids: The injected plasma case // Phys. Rev. 121, p. 26 (1961).

8. R.N. Bhargava, Negative resistance in GaP electroluminescent diodes // Appl. Phys. Lett. 14, No.6, p. 193-195 (1969).

9. K. Maeda, Double injection in GaP electroluminescent diodes // Appl. Phys. 9, No.1, p. 71-80 (1970).

10. F.P. Korshunov, G.V. Gatalskij, G.M. Ivanov, Radiation Effects in Semiconductor Devices. Minsk, Nauka i tekhnika, p. 231, 1978 (in Russian).

11. E.Yu. Brailovskij, I.D. Konosenko, V.P. Tartachnyk, Defects in GaP upon electron irradiation // Fizika i tekhnika poluprovodnikov, 9, No.4, p. 769771 (1975), in Russian. 